\title{
MÁS ALLÁ DE LA SEGURIDAD Y LA LIBERTAD La solidaridad como motor alternativo de las políticas migratorias
}

\author{
Diego Blázquez Martín \\ Universidad Carlos III de Madrid \\ Instituto de Derechos Humanos Bartolomé de las Casas
}

\begin{abstract}
In this article the author pretends to show how a security based model of migration policies do not resolve the main problems of immigration, specially irregular one, neither security issues. In this sense, the author analyzed the result statistics of the Sistema Integral de Vigilancia del Estrecho (SIVE),a complex device to control sailing in the Strait of Gibraltar. Opposite this model, it is proposed another one more "realisticn and based in a Solidarity approach which allows to justify new rights; an alternative strategy concern with the real causes of immigration, specially irregular one, as well as international security agenda issues.
\end{abstract}

KEYWORDS Immigration, security, solidarity, freedom, human rights, development.
RESUMEN En el presente trabajo se pretende poner de manifiesto como el enfoque de la regulación de flujos migratorios basado en la seguridad no resuelve los problemas que subyacen a la inmigración, especialmente la irregular, ni siquiera a los relativos a la seguridad. Para ello se analizan los resultados del Sistema Integral de Vigilancia del Estrecho (SIVE), un complejo dispositivo de control de la navegación instalado en el estrecho de Gibraltar. Frente a ese modelo, se propone otro más "realista" basado en el valor de la solidaridad y que permitiria justificar nuevos derechos. Estos derechos atajarian las causas reales de la inmigración, especialmente en el caso de la inmigración irregular, y la inseguridad internacional.

PALABRAS CLAVE Inmigración, seguridad, solidaridad, libertad, derechos humanos, desarrollo.

\section{Introducción}

Los Estados del Norte contemplan cada vez más la cuestión de la inmigración en general, pero ante todo la irregular, como un problema de seguridad interna $y$, por lo tanto, como una cuestión de índole policial, olvidando la concurrencia de otras dimensiones normativas, así como las causas globales del problema. Con suma frecuencia se enfoca la cuestión tan sólo desde la perspectiva de la seguridad nacional, poniendo en tela de juicio la prioridad de las libertades individuales. Se hace manifiesta así una frágil conciencia acerca de los derechos fundamentales, pues se está dispuesto a sacrificarlos en beneficio de una seguridad y una estabilidad convertidas en valores supremos a la hora de diseñar las politicas de inmigración. Esta actitud responde a una concepción de la inmigración utilitarista y autista, que, lejos de resolver problemas, agrava los existentes y crea otros nuevos. Por ello, cada vez se hace más patente que sólo se puede entender la inmigración desde una perspectiva que tenga en cuenta sus causas globales.
Partiendo de esa perspectiva, el propósito de este trabajo es poner de manifiesto como, al menos en el caso de la inmigración, la tensión entre libertad (de movimientos) y seguridad es una falacia, que responde a una estrategia de reducción de derechos para evitar la emancipación o mantener el sometimiento. Por el contrario, la solución de los problemas que se plantean cuando se trata la cuestión de la inmigración no tiene porque ir acompañada de manera automática de una reducción de derechos, sino, de un incremento de los mismos.

Una perspectiva realista y global muestra como las migraciones representan uno de esos uriesgos y problemas de carácter planetario" que "requieren una estrategia global tanto al interior de los Estados como en el ámbito internacional", y que justifican la existencia de unos nuevos derechos, que, asentados sobre la base de la solidaridad como la única y más razonable actitud posible para afrontar estos riesgos comunes, suelen denominarse "derechos de cuarta generación" (Rodriguez Palop, 2002, 326). Es la solución única y más razonable si, como señala Javier de 
Lucas, comenzamos por lo obvio: tomando en serio la inmigración como cuestión política que pone de manifiesto una sociedad que surge basada en el verdadero "efecto llamada" que supone la "sobreabundancia de oferta en órbita precaria, dispuesta a lo que sea por aterrizar [en el centro del mercado global]"; de manera que los desafíos que plantea la inmigración desde esta óptica exigen otras políticas "basadas en unas relaciones internacionales equitativas, y uno de cuyos instrumentos fundamentales no puede ser muy diferente de lo que conocemos como codesarrollon (de Lucas, 2004, 18 y 22).

En primer lugar, voy a abordar el modelo de control de flujos generado en torno a la idea de seguridad, cuya máxima plasmación en el ámbito español es el denominado Sistema Integral de Vigilancia del Estrecho (en adelante SIVE). Este sistema constituye un buen ejemplo de esta concepción de la regulación de flujos basada casi en exclusiva en las diversas facetas asociadas a la noción de seguridad (fundamentación, objetivo, desarrollo y consecuencias). Como veremos, ese enfoque no propicia en nada la reducción de la inmigración irregular y ni mucho menos garantiza mayores niveles de seguridad interna.

A continuación, realizaré una aproximación a un modelo mucho más complejo y más difícil de implementar que se basa en la libertad, la solidaridad y el desarrollo. Un enfoque que es el que se está intentando auspiciar por parte de las instituciones internacionales, que ven la cuestión de la inmigración en su perspectiva global. Paradójicamente, esta visión global, integral y realista de la inmigración, que une derechos y desarrollo, a veces se contempla como una concepción utópica, aunque en realidad se trata de una "utopía realista", tal como denominó John Rawls a aquellas propuestas que lejos de evadir o de disolver los problemas, se enfrentan a ellos y los resuelven (cfr. Rawls, 2001, 23-24).

\section{El modelo de "la casa a defender"}

Un buen ejemplo de lo que podriamos llamar "la orden de 'la casa a defender'» y de sus efectos lo encontramos en la política española de control de los flujos migratorios, y especialmente, en la gestión de un espacio tan complicado para las politicas migratorias como es el Estrecho de Gibraltar. Ahi se ha desarrollado el SIVE, fundado en una "lógica belicistan que confunde los problemas de la inmigración y la seguridad. Este modelo obedece, por un lado, a una concepción basada en «el control y la guarda de las fronteras») (cfr. Pérez-Diaz, Álvarez-Miranda, González-Enríquez, 2001, 19-24); y, por otro, a la visión laboral y utilitarista de la inmigración, que requiere regular los flujos en función del mercado de trabajo o de otras exigencias del sistema económico, en lo que Javier de Lucas ha llamado "un cinismo instrumental».

Esta visión se encuentra muy explícitamente en el diseño comunitario de las políticas migratorias, basadas «en un repliegue de la Unión Europea como fortaleza, el cierre de sus derechos entendidos como privilegios presuntamente amenazados por quienes acechan extra murosn, a la vez que se refuerza la idea de una Europa "cerrada sobre si misma, satisfecha de su propio bienestar y riqueza, recreada en la contemplación de su magnifica historia y de su seguridad presente" (de Lucas, 1996, 9-11).

Efectivamente, el desarrollo del SIVE se fundamenta en la política comunitaria que se pone en marcha a partir de 2001 y que pretende establecer una gestión de las fronteras externas de la Unión Europea que permita garantizar un nivel elevado y uniforme de control de las personas y de vigilancia "como condición previa a la creación de un espacio de libertad, seguridad y justicia». Dicha politica que justifica la creación de un Programa para combatir la inmigración ilegal a través de las fronteras maritimas de la Unión ${ }^{1}$.

La vinculación entre ese espacio de "libertad, seguridad y justician y el blindaje de fronteras queda perfectamente plasmado en el borrador que la Comisión presentó al Parlamento Europeo para promover en el Consejo de Salónica una politica común de control de fronteras exteriores y la consiguiente creación de una Agencia Europea para el Control de las Fronteras Exteriores, donde el primer considerando que pretende justificar esta decisión afirma "que la lucha contra el terrorismo, la delincuencia organizada, la trata de seres humanos y el tráfico de drogas hace de la seguridad un ámbito central de la UE» ${ }^{2}$. Finalmente el Reglamento 2007/2004 asume como punto de partida que "La política comunitaria relativa a las fronteras exteriores de la Unión Europea tiene por objeto establecer una gestión integrada que garantice un nivel elevado y uniforme de control y vigilancia, corolario indispensable de la libre circulación de personas en la Unión Europea y componente esencial del espacio de libertad, seguridad y justicia». 
En referencia directa a este modelo de diseño del control de la inmigración, puede leerse en la Revista de la Guardia Civil, institución encargada de este dispositivo de control de fronteras, en su Editorial del número 707 de Marzo de 2003 en la que se presentaba el SIVE, la siguiente declaración de principios: «es la inmigración irregular que trata de introducirse en nuestros países a través de todas nuestras fronteras externas, el fenómeno que adquiere una mayor relevancia [respecto de los flujos «incontrolados» de personas como problema comunitario]. Estos flujos migratorios incontrolados amenazan tanto la viabilidad de nuestras politicas de inmigración e integración como la propia estabilidad de nuestras sociedades» (la cursiva es mía). Esta visión tiende a confundirlo todo aun más al aplicar esos medios, que ya resultan poco adecuados en si mismos, a un "problema" que no se ha sabido diagnosticar correctamente.

EI SIVE, como respuesta a la inmigración irregular, es un conjunto de medios humanos y materiales cuya función es detectar cualquier movimiento en aguas del Estrecho, para posteriormente proceder a la interceptación y detención de los ocupantes de la nave en cuestión. El arsenal incluye los siguientes medios materiales: 25 estaciones sensoras en torretas provistas de radares y cámaras infrarrojas y térmicas o en furgonetas equipadas con los mismos medios; 13 radares móviles; un centro de control, desde el que agentes especializados dirigen el sistema; y un amplio cuerpo de "unidades de interceptación», para las que se prevé que en 2008 pasarán de 12 a 71, multiplicando por 6 la flota de la Guardia Civil en la zona $^{3}$, a las que no se descarta según algunas informaciones ${ }^{4}$, que incluso se incorporen dos aviones de reconocimiento.

Para todo ello, según reconocía el propio Ministerio del Interior, a lo largo de 2003 se procedió a la apertura de nuevos Centros para retener a los extranjeros irregulares en Algeciras y Tenerife, asi como a la mejora de las instalaciones de los Centros ya existentes. Estas inversiones que supusieron un coste total de 7.686.692,55 euros, junto a un presupuesto de 29.574.081,21 euros de coste directo de este SIVE durante el ejercicio $2003^{5}$, y que en conjunto, supuso un gasto total de 102 millones de euros hasta diciembre de 2004. Esta cantidad contrasta con algunas otras cifras significativas de la política exterior española de la anterior administración durante el mismo periodo. Por ejemplo, el presupuesto del SIVE hasta 2004 era cuatro veces más de lo que España invirtió en cooperación en Oriente Medio (26 millones de $€$ ), una zona en la que decía estar muy comprometida la anterior Administración, y donde, como es evidente se originan buena parte de los problemas de seguridad de la agenda ya no solo nacional o comunitaria, sino incluso mundial; pero sobre todo resalta comparándola con las cifras de la cooperación con Marruecos en el ejercicio anterior a la puesta en marcha del SIVE, puesto que es diez veces superior ( 11 millones de $€$ ).

Parece que el cambio de gobierno no está alterando sustancialmente esa política. Así, junto a los 102 millones de euros que van gastados hasta 31 de Diciembre de 2004 en el plan SIVE, se proyectan gastos por otros 130 millones en los próximos años: la implantación inicial del SIVE se calcula en 232 millones de euros.

Este complejo sistema puede tener una gran utilidad para salvar vidas, de hecho creo que es el único motivo que lo puede llegar a justificar (Abril Stoffels, 2004, 3). No obstante, también es cierto que incluso este enfoque "humanitarista», refleja a la perfección el dilema en el que, según Javier de Lucas, se ven inmersas las políticas de inmigración, encerradas entre un "cinismo instrumental», que mira la inmigración solo en clave laboral y de mercado, y un humanitarismo paternalista, que, sin refutar la visión instrumental, "enfoca la inmigración desde la ayuda al marginado" (de Lucas. 2004,15), que le salva la vida, pero luego le devuelve a su lugar de origen, sin considerar las razones que le han llevado a esa travesía.

Pero, ni por el lado "humanitarista" ni por el lado "controladorn, y ni en términos de eficiencia ni de eficacia constituye un buen sistema, puesto que a pesar de que los informes de la Guardia Civil han intentado demostrar que el sistema ha tenido una importante capacidad de prevención de la inmigración irregular, esta eficacia es relativa si hacemos un estudio un poco más detenido de lo números que presenta el Ministerio de Trabajo y Asuntos Sociales ${ }^{6}$.

En primer lugar, no es un buen sistema para luchar contra la inmigración irregular porque, tal y como la misma Relatora Especial de Naciones Unidas da por hecho en su informe sobre la situación de la inmigración en España "[32.] Las entradas irregulares se producen principalmente, según se ha informado, a través del aeropuerto internacional de MadridBarajası ${ }^{7}$, es decir, por las fronteras aéreas y terrestres.

En segundo lugar, constituye un buen ejemplo de lo que se ha denominado la guerra contra la inmigración, que tiene efectos perniciosos si lo evaluamos desde una perspectiva humanitaria. La Asociación Amigos y Familiares de Victimas 
de la Inmigración Clandestina, junto a Andalucía Acoge, ante la tragedia acaecida en Rota el 25 de octubre de 2003 en la que fallecieron 25 personas, señaló que "perseguir» a los indocumentados bajo una "lógica de guerra" no es aporta ninguna solución al problema de la inmigración irregular, y más bien lo que provoca es el agravamiento de la situación de los más vulnerables. Así, dichas organizaciones pusieron de manifiesto como, si antes del endurecimiento de las medidas de seguridad en las costas españolas las travesias que recorrian los inmigrantes en pateras eran de 14 kilómetros, ahora pueden llegar a alcanzar entre 300 y 400 kilómetros, con lo que aumentan los riesgos, incluso vemos como las rutas marítimas se han ampliado buscando costas más lejanas pero menos vigiladas lo que aumenta ciertamente el riesgo de naufragio ${ }^{8}$. De este modo se pone seriamente en peligro la vida misma de las personas que intentan migrar en esas condiciones.

Esta afirmación se puede apreciar en dos datos de las estadisticas aportadas por el Ministerio de Trabajo y Asuntos Sociales. En primer lugar, el mayor número de detenciones se han realizado en las costas más alejadas del Estrecho, donde todavía no se había desarrollado el SIVE: Málaga (donde se registra un incremento del $28 \%$ de embarcaciones), Granada (donde se incrementa ligeramente el número de embarcaciones) o incluso en lbiza (en donde ya se ha dado un primer caso).

Otro dato que pone de manifiesto la vulnerabilidad a la que se condena a los inmigrantes es el hecho del importante incremento de personas detenidas en cada viaje. Puesto que en cada viaje realizado el patrón de la patera corre un importante riesgo, intenta amortizar todo lo posible cada uno de sus desplazamientos; por lo que espacia cada vez más las singladuras, al mismo tiempo que aumenta el pasaje todo lo posible, con el consiguiente incremento de riesgo que ello supone. Esta tendencia puede apreciarse muy especialmente en el caso de Canarias, donde se reduce prácticamente a la mitad el número de embarcaciones detenidas (49\%) pero sólo se reduce un $10 \%$ el número de personas detenidas. Es decir, en la mitad de embarcaciones se detuvieron durante el año 2005 casi el mismo número de personas. Lo mismo sucede en las costas más alejadas del litoral andaluz, donde el incremento de embarcaciones va acompañado de un incremento muy superior de ocupantes detenidos: por ejemplo en Málaga, donde se habia aumentado un 30\% el número de pateras detenidas, se incrementó un $60 \%$ el número de personas detenidas; así también en Granada, donde ese pequeño $4 \%$ de embarcaciones va acompañado por un $30 \%$ de aumento de personas detenidas.

Hay un elemento final que demuestra la ineficiencia de este programa y su desconocimiento de las causas de la inmigración irregular. Resulta muy difícil medir el efecto preventivo de estas medidas, algo casi imposible de analizar de forma fehaciente. Nadie se ha atrevido a aportar datos que prueben que este mecanismo impida de hecho la inmigración y mucho menos que elimine de raiz sus causas reales. En tal caso, tan sólo se cierra o se dificulta una vía de entrada. No sabemos si los inmigrantes que pensaban cruzar el Estrecho irregularmente han decidido cancelar su proyecto migratorio, o han decidido realizarlo de otra manera (quizás a ello responda el incremento sin parangón de las pateras en Ceuta: $2760 \%$, o la presión de las fronteras terrestres de Ceuta y Melilla) y que en ese nuevo viaje lo hayan alcanzado, con lo que la supuesta eficacia preventiva desaparece, 0 al menos se pone en tela de juicio, sobre todo teniendo en cuenta que en los últimos años, especialmente los dos últimos de funcionamiento del SIVE no ha dejado de incrementarse la inmigración irregular ${ }^{9}$.

Y si tomamos como efectivos los números que se nos presentan, queda de manifiesto la señalada ineficiencia del sistema. Para reducir en un $18 \%$ la entrada ilegal por la frontera sur, lo que en términos absolutos suponen 3.501 personas, se han gastado esos 102 millones de euros, es decir, prevenir cada entrada de inmigrante irregular ha supuesto un desembolso para los contribuyentes de nada menos que de $29.134,5$ euros. Un dato que quizás merezca la pena ser conocido y discutido por la opinión pública.

Finalmente esta lógica se demuestra ineficaz y conduce a una constante reducción de derechos y de preocupación de la seguridad. Porque como las circunstancias no se han modificado y el impulso de migrar permanece, es necesario ampliar el área del SIVE porque las pateras toman otras rutas nuevas que también habrán de ser vigiladas. Por ello, cada vez el blindaje tiene que extenderse más y más, porque para que sea realmente efectivo no puede dejarse ni un centímetro de costa al descubierto. Debido a ello, recientemente el gobierno español se ha ofrecido a desarrollar la tecnología del SIVE en las costas atlánticas de Marruecos, insistiendo así en mantener esa vinculación entre seguridad e inmigración; un vínculo que se tornaba explícito por el hecho de que esta oferta la realizaran conjuntamente los Secretarios de Estado de Seguridad y de Inmigraciones y Emigración ${ }^{10}$.

ARBOR CLXXXI 713 MAYO-JUNIO [2005] 143-153 ISSN: 0210-1963 


\section{El modelo liberal: la receta del (libertad, solidaridad y desarrollo)}

\subsection{Un concepto más amplio de libertad como garantía de seguridad}

Frente a esta concepción que aborda el control de la inmigración como un problema de seguridad y le da una respuesta supuestamente técnica, se torna cada vez más evidente la urgencia de fundamentar un modelo alternativo basado primordialmente en el valor de la libertad. Para ello, es necesario situarse ante el fenómeno de la inmigración de manera realista (de Lucas, 2004, 16), y ello supone recordar que las personas que inmigran se mueven acuciados por la grave situación de desigualdad en la que se encuentra el mundo. Como ha señalado Carrillo Salcedo, al hablar de inmigración uhay que distinguir los mitos de las realidades y tomar conciencia de los efectos negativos de la globalización (...) de cada tres dólares prestados por los paises ricos, dos han de ser dedicados al pago de intereses; que el 20\% de los pobres del mundo disponen del 1,1\% del producto mundial bruto (...); que más de mil millones de personas no tienen acceso a la salud, a la educación básica o el agua potable; que dos mil millones carecen de acceso a al electricidad (...) Mientras esta injusta situación se mantenga, los flujos migratorios desde el Sur hasta el Norte, desde el mundo de la pobreza hasta el del Bienestar, serán incontenibles" (Carrillo Salcedo, 2005, 22 y 23).

En el famoso discurso de "Las Cuatro Libertades", el Presidente F.D. Roosevelt señaló a los miembros del Congreso en 1941, que "En los dias del futuro que queremos hacer cierto, buscamos un mundo basado en cuatro esenciales libertades humanas: la libertad de expresión, la libertad de conciencia; la libertad frente al miedo; y la libertad frente a la necesidad". El modelo alternativo al de seguridad se basa en esa idea amplia de Libertad. En primer lugar, porque es una libertad para todos, y no como el modelo comunitario que pretende crear un espacio interno y exclusivo de libertad. Pero, no es solo un modelo de libertad de movimientos, sino además en la libertad frente a la necesidad. Este es el enfoque que el actual Secretario General de Naciones Unidas, Kofi Annan, preconiza en su reciente informe «Un concepto más amplio de la libertad: desarrollo, seguridad y derechos humanos para todos" ${ }^{11}$.

Desde esa perspectiva, realista y comprometida con la libertad, en segundo lugar, no podemos olvidar que los problemas de inseguridad se explican en buena parte por esos problemas de desigualdad radical en la asignación de recursos.

Por otro lado, se trata de una apuesta por la libertad, ya que resulta paradójico que en estos tiempos de múltiples liberalizaciones, en estos tiempos de libre movimiento de capitales y bienes, quienes en realidad no pueden moverse libremente sean las personas. Esta situación es quizás la mejor ilustración del peligro del que nos alertaba el ilustre andaluz Fernando de los Ríos $(1975,154)$ : que la economía acabara esclavizando al hombre. Ahora se torna evidente la estrecha conexión existente entre la globalización de la economía, la emergencia de mercados de trabajo barato y las crecientes restricciones a la migración. Es precisamente frente a ese "nuevo concepto", frente a ese discurso coherentemente articulado en torno a derechos que igualan a todos los individuos, que surgirá la previsible respuesta azuzada por el temor de que se haga realidad aquella «invasión" de la "famélica legión" que anunciaba el himno de la Internacional Comunista.

En primer lugar, parece que "tal» invasión no parece que se vaya a producir ${ }^{12}$. Así lo ha puesto de manifiesto el profesor y europarlamentario Sami Nair cuando ha señalado que "La respuesta no se puede reducir a patrullas policiales en las fronterası" ${ }^{13}$, ni en la creación de controles exhaustivos en los pasos fronterizos. Por el contrario, "(...)La apertura de las fronteras a la emigración laboral parece influir más en la forma que toman las migraciones que en su importancia cuantitativan 14 .

Sin embargo, y en segundo lugar, dando por hecho que eso fuera cierto, y que nos enfrentásemos a una invasión de desposeídos, este argumento tiene una fácil contestación, que no es otra que acudir a las razones finales de esa temida invasión, y que constituyen la pobreza y la desigualdad que sufren los paises del Sur. Ferrajoli establece una muy adecuada comparación con lo sucedió en la Europa Occidental, cuando a lo largo del siglo XIX la pobreza y la desigualdad que sufrian las personas menos afortunadas planteaba una amenaza al poder $y$ al estilo de vida de unos pocos privilegiados. Estos protegían su situación privando de derechos políticos a los pobres, mientras que éstos lo que realmente demandaban eran la posibilidad real de ejercer esos derechos políticos. Fruto de esa presión resultó la universalización de los derechos políticos, así como de los derechos sociales, económicos y culturales. Quizás ese sea el papel histórico que tenga que jugar la inmigración: crecer hasta 
hacerse irresistible de manera que cedan las restricciones de derechos (cfr. Ferrajoli, 1999, 117).

Para abordar de esta manera la cuestión, tenemos que levantar todos los velos de abstracción. Y reconocer la situación que tenemos entre manos. Dos son las maneras más habituales de inmigrar ilegalmente: entrar pagando el visado de turista o infiltrándose en las fronteras. La más trágica de las inmigraciones es la que se realiza de esta segunda manera, es la inmigración de los más vulnerables, con menos recursos y con más urgencia por abandonar su pobreza, de los que caen en manos de mafias y organizaciones corruptas (cfr. Goytisolo y Nair, 2000, 15; Solanes Corella, 2002, 255).

Desde esta visión, como observó la Relatora Especial en su viaje a España, "[77]. en la realidad la inversión en medios y tecnologías, la introducción de cambios legislativos y la aplicación de políticas dirigidas a frenar la inmigración irregular no han sido suficientes para brindar a una disminución del fenómeno. La Relatora Especial observó como los migrantes terminan por intentar la entrada en el pais por otros medios mucho más peligrosos en manos de las redes, pagando en muchas ocasiones con la vida [...]n. Frente a ello, resulta casi vergonzoso que se tenga que recordar a un pais supuestamente informado que "[78] (...) las dificultades para frenar la inmigración irregular se deben a muchos factores, entre ellos la difícil situación económica y política de los paises de origen y la visión que los migrantes tienen de España y Europa como un paraiso de oportunidadesi. Por lo tanto, "84. La Relatora considera que el control de fronteras per se no asegura una gestión migratoria en orden y dignidad. En el marco de la Unión Europea, la cuestión sobre la migración debe ejecutarse en un diálogo constante con los paises de origen... que va más allá del plano laboral... El enfoque de este diálogo debe ser prevención de migración irregular y el codesarrollo en los paises de origen» ${ }^{15}$.

En este sentido, la Relatora Especial de Naciones Unidas para los trabajadores migrantes ya habia hecho énfasis de manera general en que medidas de este tipo no sólo son erróneas porque contemplan la inmigración desde un ángulo punitivo, represivo y unilateral, sino que además se han demostrado ser muy poco eficaces. Resultan asimismo tremendamente perjudiciales para las personas que migran. Por ello, en el informe se subraya "que el fenómeno de las migraciones irregulares se debería abordar mediante un nuevo concepto de la gestión de las migraciones del cual los derechos humanos son parte esencial" ${ }^{16}$. Se trata de articular un modelo que incluya estas dos dimensiones: unos derechos sobre la migración y unos derechos que se dirijan a remover las causas globales de la misma, que en el fondo radican en el subdesarrollo.

\subsection{Derechos específicos de los inmigrantes: los derechos como forma de control}

Los derechos de los inmigrantes en cierta manera tienen ya una base normativa. Sin embargo, según el status de los principales instrumentos internacionales de Derechos Humanos de la Oficina de la Alta Comisionada de Derechos Humanos (2003) ${ }^{17}$, ninguno de los estados de recepción de la inmigración ha firmado ni como estado signatario ni como estado parte la Convención internacional sobre la protección de los derechos de todos los trabajadores migratorios y de sus familiares, adoptada por la Asamblea General en su resolución 45/158, de 18 de diciembre de 1990. Esta convención sólo entró en vigor tras trece años de negociaciones y de espera el 1 de julio de 2003 gracias a la ratificación de 22 paises, para lo que tuvo que ser objeto de una larga campaña internacional de promoción del que se denominaba "el instrumento más oculto de Naciones Unidas", tal como lo tildó el Comité Directivo Internacional para la Campaña mundial para la ratificación de la Convención sobre los derechos de los trabajadores migratorios ${ }^{18}$.

Como se ha indicado anteriormente, se trataba de los estados de los que salen emigrantes, sin embargo en los cuales residen sólo unos 4,5 millones de inmigrantes, lo que representa únicamente el 2,6 por ciento del total de migrantes, mientras que el 60 por ciento de todos los migrantes, que se calcula en torno a 175 millones de personas en todo el mundo, viven en Europa y EEUU ${ }^{19}$.

El fundamento de este cuerpo normativo es que, como señalan los Considerandos primero y segundo de la Convención, la mejor forma de desalentar la contratación de inmigración irregular, y con ello la lucha contra el tráfico de personas es reconociendo más derechos a los inmigrantes en situación irregular.

"Considerando que los trabajadores no documentados o que se hallan en situación irregular son empleados frecuentemente en condiciones de trabajo menos favorables que las de otros trabajadores y que para determinadas empresas ello constituye

ARBOR CLXXXI 713 MAYO-JUNIO [2005] 143-153 ISSN: 0210-1963 
un aliciente para buscar ese tipo de mano de obra con el objeto de obtener los beneficios de una competencia desleal.

Considerando también que la práctica de emplear a trabajadores migratorios que se hallen en situación irregular será desalentada si se reconocen más ampliamente los derechos humanos fundamentales de todos los trabajadores migratorios $y_{\text {, además, }}$ que la concesión de determinados derechos adicionales a los trabajadores migratorios y a sus familiares que se hallen en situación regular alentará a todos los trabajadores migratorios a respetar y cumplir las leyes y procedimientos establecidos por los Estados interesados».

Es dificil de comprender el rechazo manifestado por los estados receptores de inmigración, pues la Convención no contempla ni reconoce realmente derechos especificos para los inmigrantes, sino que busca garantizar la igualdad de tratamiento y las mismas condiciones laborales para inmigrantes y nacionales a través de una serie de medidas de garantía, que a su vez se basan en derechos que las sociedades desarrolladas del Norte tienen reconocidos desde hace ya tiempo.

La Convención distingue sistemáticamente varios regímenes jurídicos aplicables a los trabajadores inmigrantes y sus familias. Toda la Convención se articula en torno al principio general de igualdad y no discriminación que recoge el art. 7, y que constituye en sí mismo la Parte II de la Convención, bajo el epígrafe "No discriminación en el reconocimiento de derechos" que, junto a la Parte III "Derechos humanos de todos los trabajadores migratorios y de sus familiares", constituye el régimen de derechos humanos de todos los inmigrantes trabajadores, frente a las Partes IV que determina "Otros derechos de los trabajadores migratorios y sus familiares que estén documentados o se encuentren en situación regularn, o la Parte $V$ que establece las «Disposiciones aplicables a categorias particulares de trabajadores migratorios y sus familiares". Es decir, esta Convención reconoce unos derechos comunes a todos los inmigrantes así como una serie de derechos en función de la situación administrativa.

Dentro de ese marco de respeto y protección de la dignidad humana, la convención reconoce a los migrantes los siguientes derechos, sin duda "revolucionarios" en los paises del Norte, que no han firmado esta convención:
- prevenir condiciones de vida y de trabajo inhumanas, abuso físico y sexual y tratamientos degradantes (artículos 10-11, 25),

- garantizar los derechos de los inmigrantes a la libertad de pensamiento, de expresión y de religión. (artículos 12-13),

- garantizar a los inmigrantes el acceso a la información sobre sus derechos (artículos 33),

- asegurar su derecho a la igualdad ante la ley, lo cual implica que los trabajadores migratorios estén sujetos a los debidos procedimientos, que tengan acceso a servicios legales, y que no sean sentenciados a penas desproporcionadas (artículos 16-20, 22),

- garantizar a los inmigrantes la igualdad de acceso a los servicios educativos y sociales (artículos 27-28,30).

\subsection{El desarrollo social como mecanismo de regulación de la inmigración}

En este sentido, el Capitulo IV del Plan de Acción de la Cumbre Mundial sobre Desarrollo Social de 1995 preconiza un enfoque complejo e integral respecto del problema de la inmigración irregular, y se ocupa en el párrafo 78 de dicho Plan de Acción de los intereses y las necesidad humanas básicas de los inmigrantes indocumentados, rogando a los Gobiernos en primer lugar, "cooperar para reducir las causas de la inmigración irregular, salvaguardando los derechos humanos básicos de los migrantes indocumentados, previniendo su explotación y ofreciéndoles los medios adecuados para facilitarse el acceso a la justicia de acuerdo con la legislación nacional, y castigar a los criminales que llevan a cabo tráfico de seres humanos. En segundo lugar, la Cumbre señaló a los paises de destino, a los países de tránsito y a los paises de origen que deberían cooperar de manera apropiada en la gestión de los flujos de inmigración, previniendo la inmigración clandestina y, si resultara apropiado, facilitando el retorno de los migrantes y su reintegración en sus comunidades de origen 20 .

Por lo tanto, lejos de una reducción de derechos y libertades en aras de la seguridad, un nuevo marco de derechos parece surgir de la actual situación junto a los derechos específicos. Son derechos de solidaridad que, como el derecho al desarrollo, en realidad sólo buscan una generalización y extensión de los derechos ya reconocidos. Sin embargo, resulta altamente significativo que ya en 1998 hasta una institución tan poco sospechosa como el Banco Mundial denunciara en las conclusiones de este documento que "Resulta irónico que ahora que sabemos como combatir 
la pobreza [en referencia al cambio de paradigma que desde la Convención de Viena] la ayuda al desarrollo de los Estados haya descendido a sus porcentajes más bajos de todos los tiempos»"21.

Por ello, en relación al derecho al desarrollo, no se puede olvidar la realidad y obviar que la pobreza no es un hecho en si mismo, sino que una consecuencia del sistema social y de las relaciones internacionales en que se encuentran inmersas las sociedades pobres. Igualmente, es necesario recordar que la inmigración como problema no se puede plantear de manera abstracta y general, como meros movimientos poblacionales, sino que cuando se ve como un problema es cuando hablamos de la inmigración de los países del Sur a los del Norte, y esto exige una contextualización histórica, en la que es necesario tener presente la actuación de los paises receptores sobre los emisores: "la inmigración en la actualidad no es el mundo en movimiento... es un movimiento de gentes y un mundo en movimiento que previamente ha sido trabajado, amasado con el fermente opuesto a la levadura, que ha "minorizado" a los que se hallan excluidos del sistema y ha "desvirtuado todo y a todos a quienes se trataba de dominarn (Azcona, 2002, 35). Olvidar este dato supone atribuir la responsabilidad del cambio al marginado, en vez de a la sociedad (Martín Beristain, 1999, 180), mientras que tener esto en cuenta posibilita el establecimiento del fundamento moral de un deber de cooperación al desarrollo de los Estados.

Al fin y al cabo, tal como señalara Fanon ya a mediados del siglo XX:

"Frente a ese mundo [subdesarrollado, un mundo de miseria e inhumano, lo llama Fanon] las naciones europeas se regodean en la opulencia más ostentosa. Esta opulencia europea es literalmente escandalosa porque ha sido construida sobre las espaldas de los esclavos, se ha alimentado de la sangre de los esclavos, viene directamente del suelo y del subsuelo de ese mundo subdesarrollado. El bienestar y el progreso de Europa han sido construidos con el sudor y la sangre de los negros, los árabes, los indios y los amarillos" (Fanon, 1965,88).

Por eso, en pleno proceso de descolonización Fanon afirmaba que los Estados imperialistas cometerian un "grave error y una injusticia incalificablen si se limitaran a retirarse de los territorios colonizados; por el contrario, afir- ma el mismo autor, "la reparación moral de la independencia nacional no nos ciega, no nos satisface. La riqueza de los países imperialistas es también nuestra riquezan, por lo que la ayuda a los países subdesarrollados «debe ser la consagración de una doble toma de conciencia, toma de conciencia por los colonizados de que las potencias capitalistas se la deben, y para estas, de que efectivamente tiene que pagan. Lo que espera el Tercer Mundo según Fanon, "de quienes lo han mantenido en la esclavitud durante siglos es que lo ayuden a rehabilitar al hombre, a hacer triunfar al hombre en todas partes, de una vez por todas" (Fanon, 1965, 94 y 97).

Sin embargo, como apunta Brian Barry, en la filosofia politica y jurídica de corte tradicional, «nadie cuestionaba seriamente la idea de que los límites de la justicia son los límites del estado". De tal modo que posición hegemónica no consideraría la justicia de la distribución de la riqueza en el mundo como un todo hasta muy recientemente (Barry, $1995,20)^{22}$. En mi opinión, el cambio de actitud obedecería a la introducción de la cuestión de los derechos en el discurso moral, político y jurídico: pues el carácter universalista de los derechos humanos ha permitido romper con esa restricción que circunscribia la justicia a las fronteras de los Estados.

Junto a esta disparidad de niveles de riqueza, como señala Barry, a diferencia de la Antigüedad, contamos con los medios de redistribución adecuados de manera que "la distancia no es en la actualidad una barrera con respecto a la capacidad de ayudar». Por otro lado, para Barry, una segunda razón para la uredistribución internacional es que hace solo dos siglos que los procesos de desarrollo económico desigual han abierto inmensas desigualdades" (Barry, 1995,21). De hecho, como indica Amartya Sen, uvivimos en un mundo de una opulencia sin precedentes, difícil incluso de imaginarn y sin embargo nos encontramos al mismo tiempo con unos niveles de pobreza, de sufrimiento humano que seria relativamente fácil remediar con los medios que esa creación de riqueza nos permitiria utilizar (Sen, 2000, 15).

Muestra de este abandono de la desigualdad en términos globales es la comprensión de lo que Peces-Barba denomina "El proceso de generalización», entendido como la lucha contra "unas políticas restrictivas que circunscribia su disfrute [de los derechos] a una clase social, la burguesía" (Peces Barba, 1995, 160). En este momento, sería necesario entender el "derecho al desarrollo", al menos desde su 
dimensión moral, como el derecho a la generalización o universalización de los derechos, dando lugar a lo que Rafael de Asis (2004) ha llamado ula nueva generalización de los derechos", como un proceso en el que se alcance la universalidad del disfrute de todos los derechos por todos. Porque, hoy por hoy, como señala Javier de Lucas, la razón final de toda inmigración es que upara la mayor parte de la humanidad, no tiene sentido hablar de derechos universales, sino de frustraciones" casi universales (de Lucas, 2002, 72).

Ese nuevo enfoque de la política de inmigración consiste en hacer efectivos esos derechos, para lo cual se convierte en un pilar básico el codesarrollo, entendiendo por tal, siguiendo a Goytisolo y Nair como (Goytisolo y Nair, 2000, 65-76), lo siguiente:

- facilitar el retorno, relajando el cierre de fronteras;

- convertir a los inmigrantes en "vectores de desarrollo" en su país de origen;

- crear para ello una profunda red de financiación de micro proyectos; y

- diseñar una política de cooperación al desarrollo focalizada en la inmigración como instrumento útil y también políticas de inmigración que se conviertan en instrumentos para la cooperación al desarrollo. 


\section{NOTAS}

1 Programme of measures to combat illegal immigration across the maritime borders of the Member States of the European Union. COUNCIL OF THE EUROPEAN UNION Brussels, 28 November 2003 (15445/03) № prev. doc.: 15236/03 FRONT 170 COMIX 717.

2 PROPUESTA DE RESOLUCIÓN DEL PARLAMENTO EUROPEO sobre la Comunicación de la Comisión al Parlamento Europeo y al Consejo con vistas al Consejo Europeo de Salónica relativa al desarrollo de una política común en materia de inmigración ilegal, trata de seres humanos, fronteras exteriores y retorno de residentes ilegales (COM(2003) 323 - 2003/2156(INI))

3 Cfr. el siguiente link http://www.guardiacivil.org/prensa/actividades/sive03/index.jsp

4 Vid. El País. Lunes, 11 de octubre de 2004

5 Fuente: Balance 2003 Delegación del Gobierno para la Extranjería y la Inmigración, Ministerio del Interior, Oficina de Relaciones Informativas y Sociales, Madrid 2004. P. 17.

6 Se pueden consultar en http://extranjeros.mtas.es/es/general/PATERAS_2003_Y_ 2004.pdf

7 Informe presentado por la Relatora Especial, Sra. Gabriela Rodriguez Pizarro, de conformidad con la resolución 2003/46 de la Comisión de Derechos Humanos. (E/CN.4/ 2004/76/Add.2, 14 de enero 2004). A lo que el Gobierno respondió limitándose a señalar que esa afirmación no es cierta. Sin aportar ninguna cifra que pudiera justificar su afirmación de que "La presión de entrada irregular es mucho más numerosa en las fronteras terrestres y maritimas que en las aéreas, y no es a través del Aeropuerto internacional de Barajas donde se produce principalmente». Misión Permanente de España ante la Oficina de las Naciones Unidas en Ginebra (E/CN.4/2004/G/17 de 10 Febrero 2004). P. 11.
8 Frente a esto, por cierto, es especialmente curiosa la encendida respuesta del Gobierno español que declaro a través de la Misión Permanente de España ante la Oficina de las Naciones Unidas en Ginebra que "[Punto 35] El informe de la Relatora Especial destaca el efecto que el sistema de interceptación de inmigrantes ha tenido en la búsqueda de nuevas rutas y trayectorias, que "ahora implican un trayecto más largo y peligroson. La atribución de responsabilidad al Gobierno español por la peligrosidad asumida por los migrantes que intentan entrar en el territorio español de manera ilegal es evidentemente excesiva y resulta inaceptable. Supondría querer culpar a quien pretende combatir, de acuerdo con la Ley, el tráfico ilegal y la actuación de las mafias de que éstas utilicen vías más arriesgadas para contravenir la ley". Nota verbal de fecha 29 de enero de 2004 dirigida a la Oficina del Alto Comisionado de las Naciones Unidas para los Derechos Humanos por la Misión Permanente de España ante la Oficina de las Naciones Unidas en Ginebra (E/CN.4/2004/G/17 de 10 Febrero 2004).

9 Así, personas de la reputación del profesor Carrillo Salcedo constatan como "La avalancha de extranjeros no ha sido frenada a pesar de las baterias de diques legales (...), las dos regularizaciones extraordinarias..." y, lo que a estos efectos nos interesa más "el blindaje de Ceuta y Melilla y el Sistema Integral de Vigilancia del Estrecho" (Carrillo Salcedo, 2005, 21).

10 Vid. la nota de prensa del Gabinete de Comunicación de la Secretaría de Estado de Seguridad y de Inmigración y Migraciones tras su reunión con las autoridades marroquies el pasado 25 de enero de 2005.

11 Secretaria General de las Naciones Unidas. "Un concepto más amplio de la libertad: desarrollo, seguridad y derechos humanos para todos", Informe del Secretario General de Seguimiento de os resultados de la Cumbre del Milenio. (A/59/2005).
12 Incluso así lo pone en conocimiento del Ministerio de Defensa las conclusiones de uno de los grupos de trabajo del CESEDEN, vid. Retos a la seguridad en el cambio de siglo: (armas, migraciones y comunicaciones), España . Ministerio de Defensa. Secretaría General Técnica, Madrid, 2000. Pp. 117 y 118. Igualmente en otro grupo de trabajo especifico sobre Inmigración y seguridad en el Mediterráneo, se alertan de múltiples riesgos, pero en ningún momento se hace referencia a una avalancha (vid. Inmigración y seguridad en el Mediterráneo: el caso español, Ministerio de Defensa, Madrid, 2002)

13 Sami Nair: "Los que vienen aquí», El País, Tribuna, 26-12-03.

14 Sami Nair: "Cinco ideas falsas sobre la inmigración en España» EL PAIS, Tribuna. 16-05-2002.

15 Informe presentado por la Relatora Especial, Sra. Gabriela Rodríguez Pizarro, de conformidad con la resolución 2003/46 de la Comisión de Derechos Humanos. (E/ CN.4/2004/76/Add.2, 14 de enero 2004) Pp. 20 y 21.

16 Vid. Informe de la Relatora Especial, Da. Gabriela Rodríguez Pizarro, conforme a la Res. 2002/62 de la Comisión de Derechos Humanos (E(CN.4/2003/85, 30 de Diciembre de 2002).

17 Se puede obtener versión electrónica en http://www.unhchr.ch/pdf/reportsp.pdf (pendiente de últimas actualizaciones) que se pueden conocer por medio de la pagina web del Comité en apoyo de la Convención: Se puede ver el sitio Oficial del Comité Directivo Internacional para la Campaña mundial para la ratificación de la Convención sobre los derechos de los trabajadores migratorios en http://www.migrantsrights.org

18 Campaña que incluyó una Resolución de la Comisión de Derechos Humanos de 1992 (Res. 1992/81), una Resolución del Consejo Económico Social de 22 de febrero de 1994, así como una mención en el Programa de Acción de la Conferencia de Derechos Humanos de Viena de 1993, todos ellos instando a la firma y ratificación de la Convención. 


\section{BIBLIOGRAFÍA}

19 Fuente: Informe sobre Migración Internacional 2002, Naciones Unidas (Departamento de Asuntos Económicos y Sociales, División de Población) Se pueden encontrar los resúmenes de estas estadisticas en el documento de difusión de esta convención «La Convención de las Naciones Unidas sobre los derechos de los inmigrantes" UNESCO, Ginebra 2003 (pp. 4 y 7).

20 Programme of Action of the World Summit for Social Development. World Summit for Social Development Agreements (Copenhagen, 1995), se puede leer el texto de los Acuerdos en http://www.un.org/ esa/socdev/wssd/agreements/ poach4.htm

21 Development and Human Rights: the Role of the World Bank. World Bank, Washington, 1998. P. 30. Respecto al caso de la política de cooperación al desarrollo en el Norte de África por parte de la Unión Europea y los Estados Miembros, cfr. Nair, 1998.

22 Para Brian Barry el primer estudio que toma en serio esta cuestión desde tal perspectiva es el realizado por Charles S. Beitz (1979)
Abril Stoffels, Ruth (2004): "La regulación jurídica de la asistencia humanitaria en los conflictos armados: logros y lagunas”, en IRRC, Vol. 86, No 855

Azcona, Jesús (2002): "Las barreras de las culturas identitarias: migración y limites sociales", en Identidades culturales y Derechos Humanos (ed. Manuel Calvo), Dykinson, Madrid.

Barry, Brian (1995): Teorías de la Justicia (trad. C. Hidalgo) Gedisa, Barcelona.

Beitz, Charles S. (1979): Political Theory and International Relations, Princeton U.Press, Princeton.

Carrillo Salcedo, Juan Antonio (2005): "La entrada de Inmigrantes Irregulares" en A.A.V.V. Inmigración Irregular y Derecho (ed. Alejandro del Valle Y Miguel Ángel Acosta), Servicio de Publicaciones de la U. de Cádiz, Cádiz.

Ceseden (2000): Retos a la seguridad en el cambio de siglo: (armas, migraciones y comunicaciones), España. Ministerio de Defensa. Secretaria General Técnica, Madrid.

Ceseden (2002): Inmigración y seguridad en el Mediterráneo: el caso español, Ministerio de Defensa, Madrid.

De Asis Roig, Rafael (2004): "La nueva generalización de los derechos", ponencia presentada en el Seminario Una discusión sobre la universalidad de los derechos humanos y la inmigración (13 de Mayo 2004), organizado por la Cátedra Norberto Bobbio de Igualdad y no Discriminación.

De Los Ríos, Fernando (1975): Escritos sobre Democracia y Socialismo (ed. Virgilio Zapatero) Taurus, Madrid.

De Lucas, Javier (2004): "Como introducir el principio de justicia en las políticas de inmigración", en Laura Miraut (ed), Justicia, Migración y Derecho, Dykinson, Madrid.

De Lucas, Javier (1996): Puertas que se cierran. Europa como fortaleza. Icaria, Barcelona.

De Lucas, Javier (2002): "La exclusión como negativo de los derechos", Concepción Ortega y $\mathrm{M}^{\mathrm{a}}$ José Guerra (eds.), Globalización y neoliberalismo: ¿un futuro inevitable?, Nobel, Oviedo.

Fanon, Frantz (1965): Los condenados de la tierra (trad. J. Campos), FCE, México.
Ferrajoli, Luigi (1999): Derechos y Garantias: la ley del más débil (trad. Perfecto Andrés Ibáñez y Andrea Greppi), Trotta, Madrid.

Goytisolo, Juan y Nair, Sami (2000): El Peaje de la vida. Integración o rechazo de la inmigración en España. Aguilar, Madrid.

Martín Beristain, Carlos (1999): Reconstruir el tejido social, Icaria-Antrazyt, Barcelona.

Nair, Sami (1998): Las Heridas Abiertas (trad. Maria Cordón), Santillana, Madrid.

Peces- Barbas, Gregorio (1995): Curso de Derechos Fundamentales, BOE, Madrid.

Pérez-Díaz, Victor; Álvarez-Miranda, Berta y González-Enriquez, Carmen (2001): España ante la inmigración, Fundación La Caixa, Barcelona.

Rawls John (2001): El derecho de gentes y "una revisión de la idea de razón pública", Paidós, Barcelona.

Rodríguez Palop, María Eugenia (2002): La nueva generación de Derechos Humanos. Origen y justificación. Dykinson, Madrid.

Sen, Amartya (2000): Desarrollo y Libertad (trad. E. Rabasco y Luis Toharia), Planeta, Barcelona.

Solanes Corella, Ángeles (2002): "La situación jurídica del inmigrante irregular en Españan, en Natividad Fernández y Manuel Calvo (eds.), Inmigración y derechos, Mira, Zaragoza.

World Bank (1998): Development and Human Rights: the Role of the World Bank. World Bank, Washington. 